\title{
Editorial
}

\section{Integral and Differential Systems in Function Spaces and Related Problems}

\author{
Ti-Jun Xiao, ${ }^{1}$ James H. Liu, ${ }^{2}$ and Hong-Kun $\mathrm{Xu}^{3}$ \\ ${ }^{1}$ School of Mathematical Sciences, Fudan University, Shanghai 200433, China \\ ${ }^{2}$ Department of Mathematics and Statistics, James Madison University, Harrisonburg, VA 22807, USA \\ ${ }^{3}$ Department of Applied Mathematics, National Sun Yat-sen University, Kaohsiung 80424, Taiwan
}

Correspondence should be addressed to Ti-Jun Xiao; xiaotj@ustc.edu.cn

Received 3 June 2013; Accepted 3 June 2013

Copyright (C) 2013 Ti-Jun Xiao et al. This is an open access article distributed under the Creative Commons Attribution License, which permits unrestricted use, distribution, and reproduction in any medium, provided the original work is properly cited.

As has been seen, integral and differential systems in function spaces are ideal mathematical models in many applied problems stemmed from the real world. They have in recent years been major objects of investigations with fast increasing interest. This special issue is dedicated to the dissemination of current significant progresses and new trends in this field.

This issue is composed of papers that emphasize different aspects of the theory of the integral and differential systems in function spaces and related issues. The topics addressed by these published papers in the special issue include the stability of delay differential systems; the Wiener product on a bosonic Connes space associated to a bilaplacian and the formal Wiener chaos on the path space; multiplicative and additive perturbation of convoluted $C$-regularized operator families, convoluted $C$-cosine operator families, and convoluted $C$ semigroups related to the differential equations in Banach spaces; the local Gevrey regularity of the solutions of the linearized spatially homogeneous Boltzmann equations; the boundedness of some rough bilinear fractional integral on Morrey spaces and modified Morrey spaces; the global bifurcation of positive solutions for semilinear elliptic equations with asymptotically linear function on a unit ball; hybrid gradient-projection algorithm for solving constrained convex minimization problems with generalized mixed equilibrium problems; the pointwise estimates for the sharp function of the maximal multilinear commutators and maximal iterated commutator generalized by $m$-linear Calderón-Zygmund singular integral operator; and the existence of nontrivial solutions of a quasilinear elliptic equation.

\section{Acknowledgment}

We would like to thank all the authors who submitted manuscripts for consideration in this special issue and regret that many could not be accepted. Special thanks go to all the reviewers for their valuable comments, criticism, and suggestions.

Ti-Jun Xiao

James H. Liu

Hong-Kun Xu 


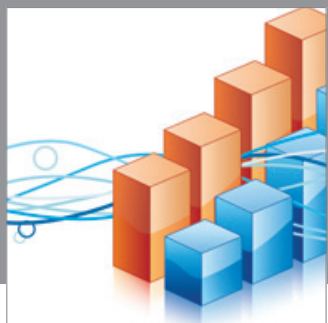

Advances in

Operations Research

mansans

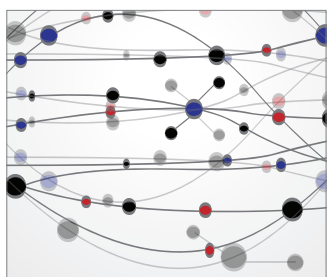

The Scientific World Journal
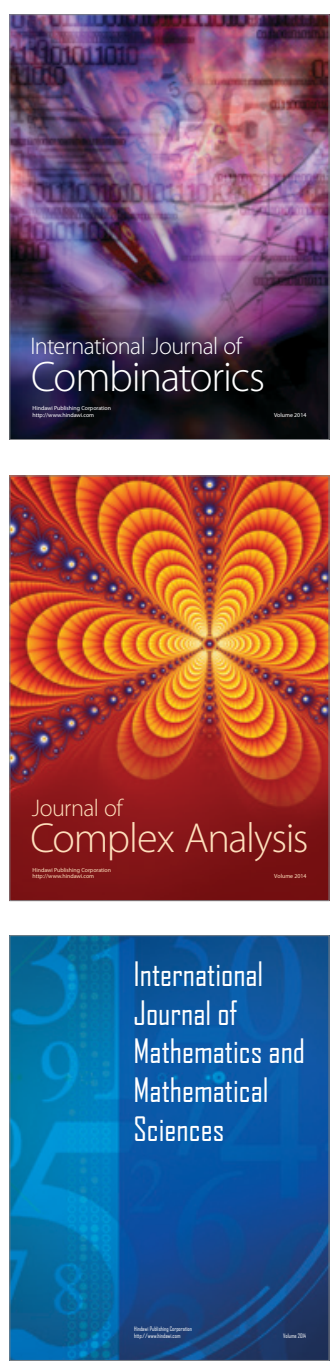
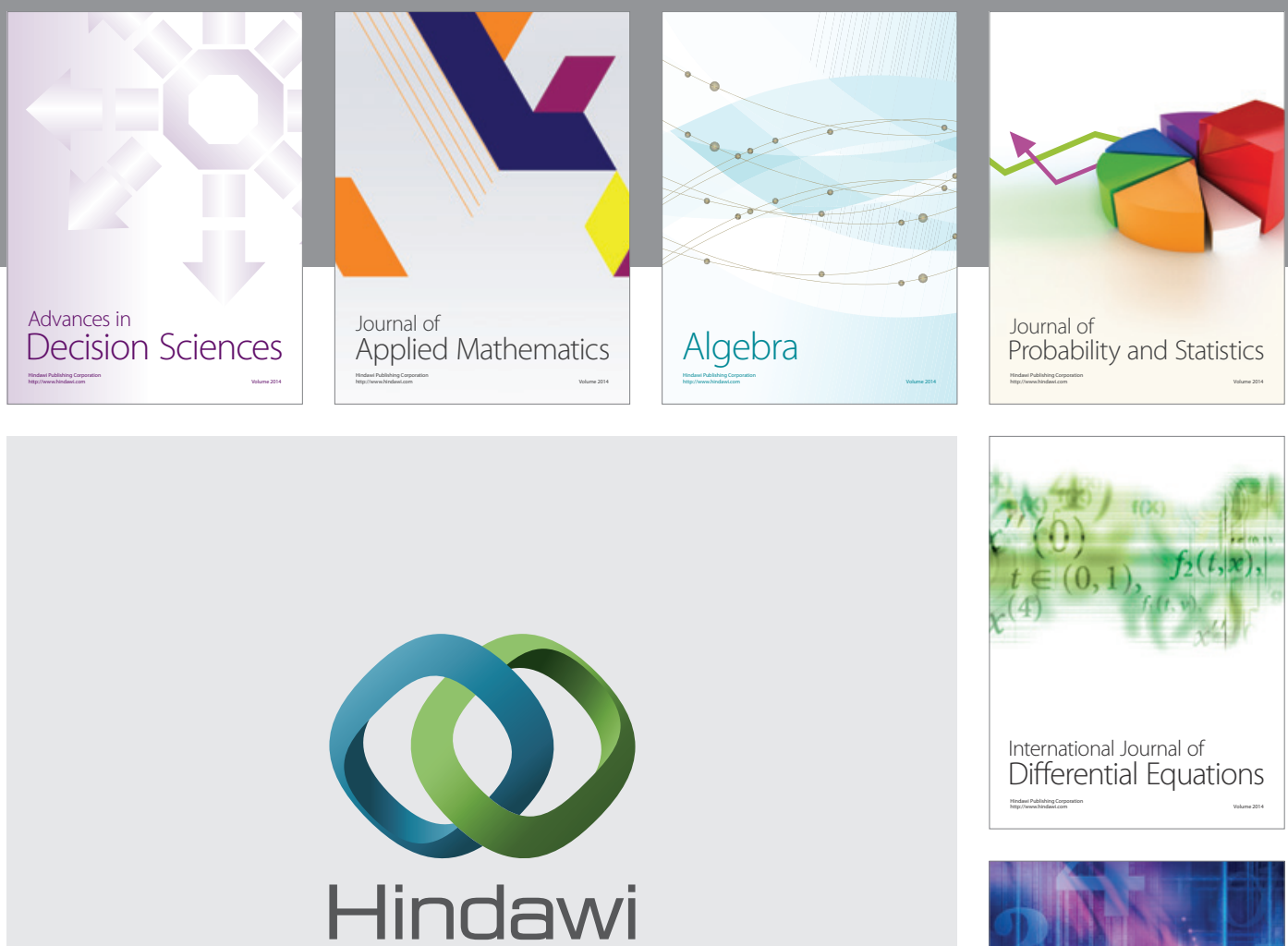

Submit your manuscripts at http://www.hindawi.com
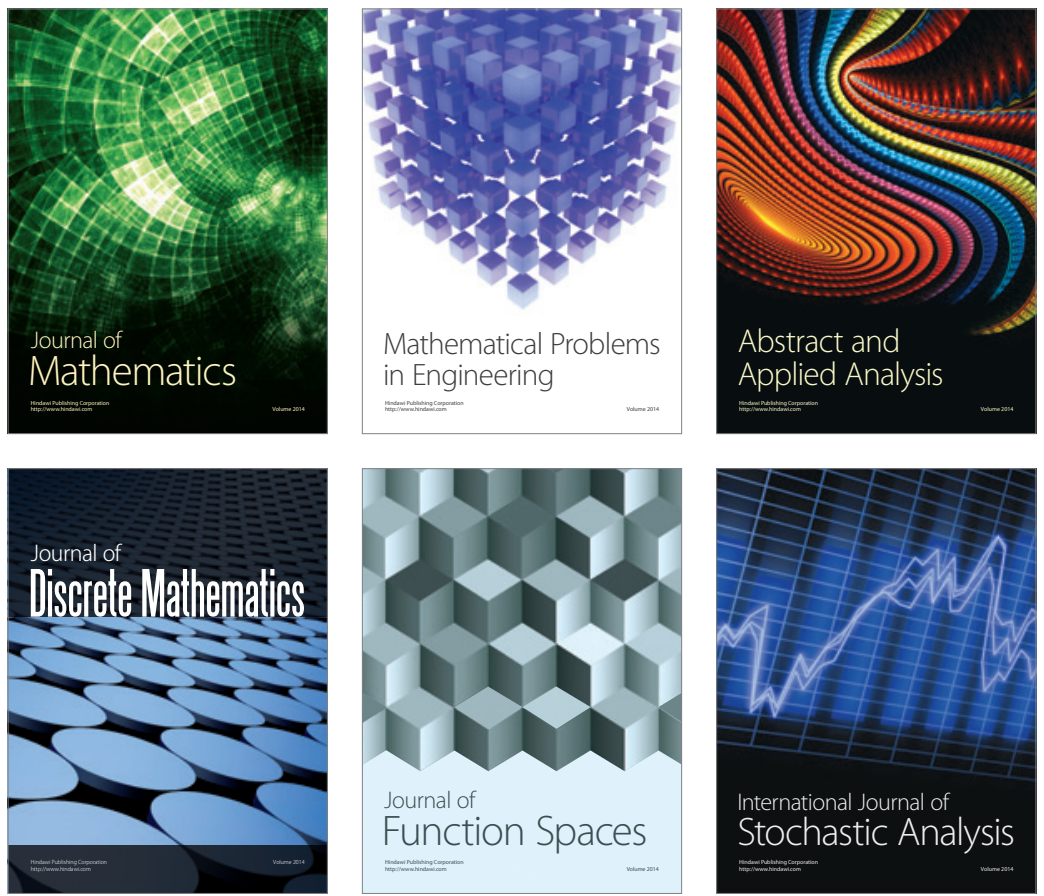

Journal of

Function Spaces

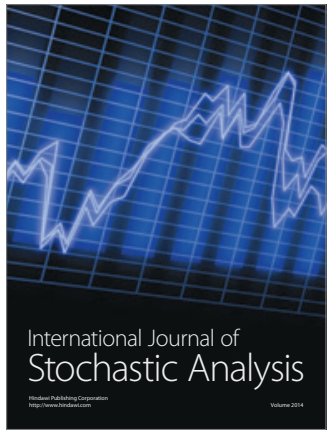

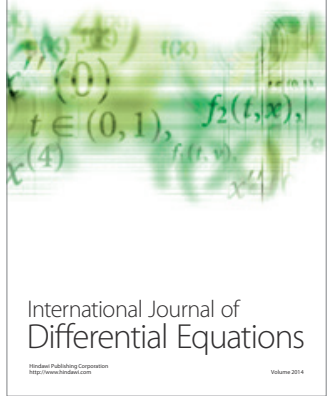
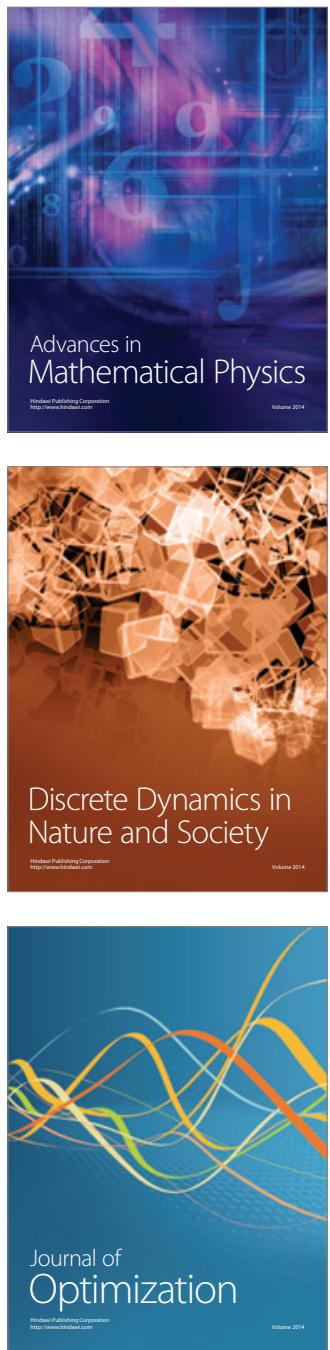